\title{
Research Paper: The Role of Thwarted Belongingness, Perceived Burdensomeness, Self-Efficacy and Ego Strength in Predicting Suicidal Ideation of Nurses
}

\author{
Esmaeil Sadri Damirchi ${ }^{*}$ (D), Nasim Zakibakhsh Mohammadi², Seyed Mohammad Basir Amir ${ }^{1}$ (D)
}

1. Department of Counseling, Faculty of Education and Psychology, University of Mohaghegh Ardabili, Ardabil, Iran.

2. Department of Psychology, Faculty of Education and Psychology, University of Mohaghegh Ardabili, Ardabil, Iran.

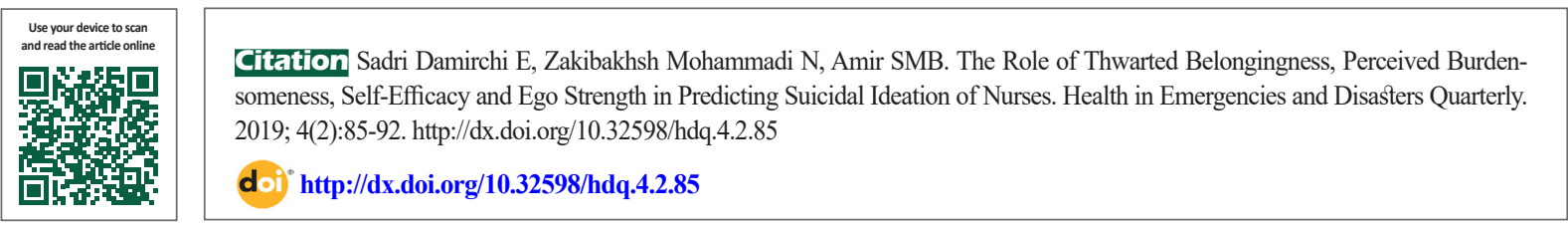

\section{(i) \$}

Article info:

Received: 10 Aug 2018

Accepted: 05 Dec 2018

Available Online: 01 Jan 2019

\section{Keywords:}

Thwarted Belongingness, Perceived Burdensomeness, Self-Efficacy, Ego Strength, Suicidal Ideation

\section{A B STRACT}

Background: More than a million people worldwide die by suicide every year, the risk of committing suicide in healthcare related occupations, especially nursing, is much higher than other occupations. The current study aimed at investigating the role of the Thwarted Belongingness (TB), Perceived Burdensomeness (PB), Self-Efficacy (SE), and Ego Strength (ES) in predicating Suicidal Ideation (SI) in nurses.

Materials and Methods: In the current descriptive-correlational study, convenience sampling method was employed to select 120 nurses of hospitals in Ardabil, Iran. The participants completed the Interpersonal Needs Questionnaire (INQ), General Self-Efficacy Scale (GSES), Psychology Inventory Ego Strengths (PIES), and Beck Scale for Suicide Ideation (BSSI). The collected data were analyzed by descriptive statistics indices, Pearson correlation analysis, and multivariate regression with SPSS V. 23.

Results: The obtained result showed a significant and positive relationship between Thwarted Belongingness and Perceived Burdensomeness, and Suicidal Ideation as well as a significant and negative relationship between Self-Efficacy and Ego Strength, and Suicidal Ideation. Also, the result of the multivariate regression showed that Thwarted Belongingness, Perceived Burdensomeness, Self-Efficacy, and Ego Strength predictability can account for $46 \%$ of Suicidal Ideation. Also, the result showed that Perceived Burdensomeness could explain and predict Suicidal Ideation better than other variables.

Conclusion: Generally, Thwarted Belongingness and Perceived Burdensomeness, as two interpersonal factors, and Self-Efficacy and Ego Strength, as two intrapersonal factors, can predict Suicidal Ideation of nurses. Possession of the sense of Thwarted Belongingness leads to a feeling of loneliness, and perceiving burdensomeness leads to a feeling of self-hatred, and ultimately, the combination of these two structures leads to the formation of Suicidal Ideation. Also, poor SelfEfficacy leads to negative judgment of abilities and the low Ego Strength uses inappropriate defense mechanisms such as denial and suppression, and ultimately, the combination of these two forms Suicidal Ideation. Therefore, providing the training to improve interpersonal relationships in order to reduce the felling of Thwarted Belongingness and Perceived Burdensomeness as well as individual counseling to improve Self-Efficacy and Ego Strength can be effective in reducing Suicidal Ideation and suicide attempt in nurses.

\footnotetext{
* Corresponding Author: 


\section{Introduction}

uicide is defined as the act of deliberately ending one's own life [1]. More than a million people worldwide die by suicide every year [2]. Suicidal behaviors and ideation are among the most common reasons for referral to the emergency department [3]. It is noteworthy that $36-39 \%$ of the individuals with suicide attempts or died by suicide were in contact with the emergency department 12 months prior to suicide [4]. Research results show that the risk of committing suicide in the medical community as well as other health care workers, especially nurses, is much higher than other occupations [5]. Despite the high rate of suicide in the nursing community, the number of studies conducted in this area is very limited [6].

A number of studies in several countries shown an increased risk of suicide attempts among nurses, but very little information is provided in relation to the causes of suicide attempts by nurses [7]. In a study on the factors related to suicide among doctors and nurses, it was observed that suicide attempt was higher among nurses than doctors and the general population. The reasons for this difference were reported as familiarity of nurses with various suicidal drugs, occupational stress, mental exhaustion as a result of heavy work, and depression due to the effects of work environment [8]. Osafo et al. (2018) investigated the attitudes of physicians and nurses towards suicide [9]. Hawton et al. (1999) compared the risk of suicide in medical groups and general population, and reported that the risk of suicide was higher in nurses [10].

Various reasons and perspectives are mentioned to explain suicidal behaviors. However, there are very few experimental and systematic studies on suicide. One of the reasons is the lack of a structured model to explain this behavior. To fill this gap, Joiner presented interpersonal-psychological theory of suicidal behavior. According to this theory, an individual will not die by suicide unless she/he has both the desire to die by suicide and the ability to do so. Regarding the desire to commit suicide, this theory states two inter-organizational constructs; Thwarted Belongingness (TB) and Perceived Burdensomeness (PB). TB is the experience that one is alienated from others, or sense of low belongingness to groups and to oneself.

Individuals with $\mathrm{TB}$ do not consider themselves as an integral part of a family, circle of friends, or other valued group. $\mathrm{PB}$ is the sense that one's existence places a burden on family, friends, and/or society. This sense produces the idea that the person's death is better for his/her family, friends, and society [11]. Ma et al. (2016) reported that of 12 studies on examining the effects of TB and PB on suicide ideation and attempt, eight studies showed their significant effect, and in four studies, the reported effect was not significant; of which only two studies showed effective results. Also, among 21 studies on testing correlation between acquired ability for suicide attempt and ideation, 12 were significant and nine were not significant [1].

The research background indicates the relationship of TB and PB with suicide attempt and ideation in different groups; but so far, no study is conducted to investigate the relationship between these variables in the nursing community. Another concept that attracts the attention of many researchers to predict suicide ideation is the concept of "Self-Efficacy". This concept was introduced by Albert Bandura as the social-cognitive theory. According to him, it is one's belief in one's ability to organize and execute courses of action in order to achieve the desired goals. He also believed that Self-Efficacy was one of the most important factors regulating human behavior [12].

A study examined the moderating role of Self-Efficacy on Suicidal Ideation in Iran. According to this study, although depression is always emphasized as an important part in the underlying structure of Suicidal Ideation and attempt, Suicidal Ideation is much less likely experienced, even at the highest levels of depression, in individuals with high levels of Self-Efficacy [13]. In the study by Kobayashi et al. (2015) Self-Efficacy, depression, and demographic factors were reported as predictors of Suicidal Ideation [14]. Ammentorp et al. (2007) indicated the effect of training in communication skills on medical nurses' Self-Efficacy [15].

Yang (2011) suggested a need to develop programs for nurses to increase Self-Efficacy [16]. In Iran, there are also some studies on the relationship of Self-Efficacy with clinical decision-making [17], social health [18], and conflict management strategies of nurses [19]. However, with regard to the confirmation of Self-Efficacy's association with Suicidal Ideation in previous studies, a research is not yet conducted to examine the relationship between these two variables among nurses. The "Ego Strength" is another concept that its effect on Suicidal Ideation and behavior is less considered, and the scholars are less concerned about it in many psychological disorders and impulsive behaviors. In psychoanalytic theory, ego is one of the elements of personality, and when an individual experiences anxiety, if it becomes overwhelming, "ego" tries to control the situation by employing defense mechanisms.

Vaillant, as one of the theorists in the field of defense mechanisms, believes that compromised defenses are linked 
to physical health, life satisfaction, quality of friendship and mental health, and the greater the strength of ego, the more developed defense mechanisms are used by the person. In addition, Ego Strength can improve the individual's flexibility in using defense mechanisms [20]. In this regard, and considering that with reduced Ego Strength the immature levels of defense mechanisms (e.g. denial, displacement, projection, and repression) are emerged, and according to previous studies such as that of Plutchik et al. (1995) (reporting a significant negative relationship between Ego Strength and the risk of suicide) [21].

Apter et al. (1997) (showing that the defense mechanisms of denial, displacement, and repression had correlation with suicidal behavior) [22], Yang (2015) (suggesting that an ego-resiliency and work environment have correlation with job satisfaction of nurses) [23], and Lee et al. (2015) (showing the effect of emotional intelligence and ego-resiliency on interpersonal relationship of nurses), it can be argued that suicide attempts and Suicidal Ideation are higher in nurses with lower Ego Strength. However, no study was found on the relationship between Ego Strength and Suicidal Ideation among nurses [24].

According to the World Health Organization (WHO) that included suicide as one of the leading causes of death in the world, and given that a number of studies reported the higher risk of suicide in health care workers including nurses, and due to the very limited studies worldwide, including Iran, regarding the factors influencing the Suicidal Ideation and attempt among nurses, further research is needed. Moreover, due to the lack of studies on examining the relationship of TB, BP, Self-Efficacy, and Ego Strength with Suicidal Ideation in nurses, there is a need for research in this area. More knowledge about the role of these variables in predicting Suicidal Ideation can help to improve the treatment used to reduce suicide attempts in nurses. The current study aimed at assessing the relationship of TB, BP, Self-Efficacy, and Ego Strength with Suicidal Ideation in nurses to examine which of them can predict Suicidal Ideation in nurses.

\section{Materials and Methods}

\section{Study population and samples}

The current study had a descriptive-correlational design. TB, BP, Self-Efficacy, and Ego Strength were considered as predictor variables and Suicidal Ideation as criterion variable. Study population consisted of all nurses working in hospitals located in Ardabil, Iran, in 2017 among which 120 were selected as the study samples using convenience sampling method. To analyze data, descriptive statistics (mean and standard deviation) and statistical tests (Pearson correlation and multiple regression analysis) were used with SPSS V. 23.

\section{Data collection tools}

Psychosocial Inventory of Ego Strengths (PIES)

This questionnaire was developed by Markstrom et al. (1997) [25]. It has 64 items measuring eight subscales of hope, will, purpose, competence, fidelity, love, care, and wisdom. Items are scored on a five-point Likert scale ranged from 5 (describes me very well) to 1 (does not describe me well). High scores indicate high Ego Strength. Its reliability using Cronbach's alpha is reported 0.68. The reliability of its Persian version is reported 0.91 and its split-half reliability is 0.77 [26].

\section{Interpersonal Needs Questionnaire (INQ)}

It was developed by Van Orden (2009) and has 25 items measuring the two subscales of PB and TB. The items are scored based on a seven-point Likert scale [27]. The first 15 items assess PB and the other 10 are related to TB. This questionnaire has a good internal consistency with a Cronbach's alpha of 0.88 [28]. For its Persian version, Cronbach's alpha is reported 0.83 [29].

\section{General Self-Efficacy (GSE)}

This scale was developed by Sherer et al. (1982) It has 17 items measuring general Self-Efficacy of individuals and is scored based on a five-point Likert scale. Sherer et al. (1982) reported internal reliability of this instrument as $\alpha=0.76$ [30].

\section{Beck Scale for Suicide Ideation (BSSI)}

It is a tool to examine suicidal intent in patients. It is a 19 item instrument developed by Beck in 1961 and is scored based on a three-point Likert scale from 0 to 2 . The total score ranges from 0 to 38 . This scale has high reliability. The reliability of the Persian version of BSSI is $\alpha=0.9$ based on Cronbach's alpha and 0.74 based on test-retest [31].

\section{Results}

Of the 120 subjects, 90 (75\%) were female, and $30(25 \%)$ male, with the mean age of 33.69 years. Regarding their education, 98 (81.7\%) had bachelor's degree and 22 (18.3\%) master's degree. Table 1 shows the mean and standard deviation of scores for the study variables. In order to test the normality of data distribution, the Kolmogorov-Smirnov 
Table 1. Mean \pm SD of scores for TB, BP, SE, ES and SI

\begin{tabular}{cc}
\hline Variable & Mean \pm SD \\
\hline TB & $30.87 \pm 7.597$ \\
PB & $52.67 \pm 11.573$ \\
SE & $39.72 \pm 9.219$ \\
ES & $189.37 \pm 18.821$ \\
SI & $7.12 \pm 3.748$ \\
\hline & |llealth in \\
\hline Emergencies and [D]isasters [Oluarterly
\end{tabular}

TB: Thwarted Belongingness; PB: Perceived Burdensomeness; SE: Self-Efficacy; ES: Ego Strength; SI: Suicidal Ideation

test was used. According to Table 2, $\mathrm{P}>0.05$; hence, the assumption of normality is met.

Table 3 shows the Pearson correlation test results. As can be observed, Suicidal Ideation has a significant positive relationship with TB $(r=0.49)$ and $\mathrm{PB}(\mathrm{r}=0.59)$, and a significant negative relationship with Self-Efficacy $(\mathrm{r}=-0.44)$ and Ego Strength $(\mathrm{r}=-0.37)(\mathrm{P}<0.001)$. Multiple regression analysis results showed that TB, BP, Self-Efficacy, and Ego Strength variables had the ability to predict $46 \%$ of changes in Suicidal Ideation, whereas PB was the most powerful predictor of Suicidal Ideation (Table 4).

\section{Discussion}

The current study aimed at examining the role of TB, BP, Self-Efficacy, and Ego Strength in predicting Suicidal Ideation of nurses. The obtained results indicated that Suicidal Ideation of nurses had significant positive relationship with TB and BP. The results were consistent with those of the Rashid et al. (2016) [1], Bryan (2011) [32], O'Keefe et al. (2014) [33], and Hill and Pettit (2012) [34].

The current study results also showed that PB was the strongest predictor of Suicidal Ideation in nurses,

Table 2. Normality test between study variables

\begin{tabular}{ccc}
\hline Variable & K-S Test & $\mathbf{P}$ \\
\hline TB & 1.715 & 0.066 \\
PB & 1.319 & 0.062 \\
SE & 0.740 & 0.645 \\
ES & 0.655 & 0.784 \\
SI & 1.349 & 0.078 \\
\hline K-S test: Kolmogorov Smirnov & & 1Hlealth in \\
Emergencies and [D]isasters [Oluarterly
\end{tabular}

Table 3. Correlation coefficients of the study variables

\begin{tabular}{|c|c|c|c|c|c|}
\hline Variables & TB & PB & SE & ES & SI \\
\hline TB & 1 & & & & \\
\hline PB & $0.49 *$ & 1 & & & \\
\hline SE & $-0.30 *$ & $-0.43^{*}$ & 1 & & \\
\hline ES & $-0.35^{*}$ & $-0.07 *$ & $0.06^{*}$ & 1 & \\
\hline $\mathrm{SI}$ & $0.29^{*}$ & $0.59 *$ & $-0.44 *$ & $-0.37^{*}$ & 1 \\
\hline
\end{tabular}


Table 4. Regression coefficients for testing the predictability of SI by TB, BP, SE, and ES variables

\begin{tabular}{|c|c|c|c|c|c|}
\hline \multirow{2}{*}{ Variables } & \multicolumn{2}{|c|}{ Unstandardized Coefficient } & \multirow{2}{*}{$\begin{array}{c}\text { Standardized Coefficient } \\
\text { Beta }\end{array}$} & \multirow{2}{*}{$t$} & \multirow{2}{*}{ Sig. } \\
\hline & B & Std. Error & & & \\
\hline Constant & 12.579 & 4.327 & & 7.503 & 0.000 \\
\hline TB & 0.309 & 0.231 & 0.153 & 4.074 & 0.006 \\
\hline PB & 0.852 & 0.413 & 0.293 & 8.973 & 0.000 \\
\hline SE & 0.422 & 0.297 & -0.192 & -2.119 & 0.013 \\
\hline ES & 0.062 & 0.098 & -0.031 & -1.346 & 0.091 \\
\hline
\end{tabular}

which was in agreement with the results of Rashid et al. (2016) where scholars reported that PB was a strong predictor of suicidal behavior [1]. In this regard, it can be claimed that interpersonal-psychological theory of suicidal behavior presented appropriate explanation for the causes and interpersonal factors of suicidal thoughts and behavior, and the theory could predict the tendency of nurses to commit suicide.

Results of the current study also showed that Suicidal Ideation of nurses had a significant negative correlation with Self-Efficacy and Ego Strength; regarding Self-Efficacy variable, the result was consistent with the findings of Kobayashi et al. (2015) [14], Nabavi et al. (2017) [35], and Valois et al. (2015) [36]. The high level of Self-Efficacy has a positive effect on mental health and its low level negatively affects self-esteem, and also, it is effective on the severity of depression and anxiety; and on the other hand, depression, anxiety, and low self-esteem can increase Suicidal Ideation; therefore, it can be concluded that low levels of Self-Efficacy can increase suicidal thoughts.

Regarding Ego Strength variable, the result was consistent with the findings of Plutchik et al. (1995), and Apter et al. (1997) [21, 22]. According to the fact that by increased Ego Strength, people use more mature defense mechanisms, and with its reduction, people use immature defense mechanisms such as denial, displacement, projection, and repression, and considering that previous studies reported positive correlation between immature defense mechanisms and suicide [22], the relationship between Ego Strength and suicide can be deduced.

The current study had a correlational design. Thus, there was limitation for intergroup comparison and generalization of results to other communities or other cities data. According to previous reports regarding high rate of suicide attempt among nurses in comparison with other occupations, and based on the findings of the current study, it is suggested that nursing authorities should plan for early intervention to prevent Suicidal Ideation and subsequently suicidal attempts as an emergency for critical occupations, and prevent the development of suicidal thoughts and behaviors in at-risk nurses using psychological and educational intervention programs and holding workshops in order to reduce the underlying factors such as TB and PB and increasing their Self-Efficacy and Ego Strength.

It is recommended that with regard to the difficult conditions of the nursing career, which needs high responsibility, and considering the emphasis and strategy of the WHO in utilizing counseling and psychological services to strengthen personal relationships and beliefs, and learning positive coping skills, nurses should reduce TB and PB senses and improve Self-Efficacy and Ego Strength in themselves. Furthermore, longitudinal studies are recommended on other populations and using other techniques to achieve more accurate results.

\section{Conclusion}

According to the reports of previous studies on the high rate of suicide among nurses on one hand, and limited number of international studies on the factors associated with the formation of suicidal thoughts and behavior in nurses, and the lack of a national study in this area, the current study aimed at investigating interpersonal (TB and $\mathrm{PB}$ ) and intrapersonal (Self-Efficacy and Ego Strength) factors of this behavior in order to raise awareness, and thus identifying several factors associated with this irreparable phenomenon, and thereby reducing its incidence rate among nurses.

Since suicide is one of the key issues in the psychology of the crisis and the first step in crisis management is preparation, the current study tried to help specialists in crisis intervention to educate and prevent suicide attempts 
and make psychological readiness for exposure before occurrence by identifying a number of suicide-related factors among nurses. It was concluded that both interpersonal and intrapersonal factors had considerable role in Suicidal Ideation. Therefore, in order to prevent and treat this behavior, both interpersonal and interpersonal problems should be considered.

\section{Ethical Considerations}

\section{Compliance with ethical guidelines}

Participants were not obliged to complete the questionnaires, and they were assured of the confidentiality of their information.

\section{Funding}

This research did not receive any specific grant from funding agencies in the public, commercial, or not-forprofit sectors.

\section{Authors contributions}

All authors contributed in preparing this article.

\section{Conflict of interest}

The authors declared no conflict of interest.

\section{References}

[1] Rashid S, Kiani A, Khorramdel K. [The relationship between perceived social support, Perceived Burdensomeness and Thwarted Belongingness with suicidal behavior in college students (Persian)]. Pajoohande. 2016; 21(4):192-8.

[2] Odom-Forren J. Suicide and nurses. Journal of Perianesthesia Nursing. 2018; 33(4):363-5. [DOI:10.1016/j.jopan.2018.06.097] [PMID]

[3] Ceniti AK, Heinecke N, McInerney SJ. Examining suicide-related presentations to the emergency department. General Hospital Psychiatry. 2018; pii:S0163-8343(18):30072-0. [DOI:10.1016/j. genhosppsych.2018.09.006] [PMID]

[4] Leavey G, Rosato M, Galway K, Hughes L, Mallon S, Rondon J. Patterns and predictors of help-seeking contacts with health services and general practitioner detection of suicidality prior to suicide: A cohort analysis of suicides occurring over a two-year period. BMC Psychiatry. 2016; 16:120. [DOI:10.1186/s12888-0160824-7] [PMID] [PMCID]

[5] Alderson M, Parent-Rocheleau X, Mishara B. Critical review on suicide among nurses. Crisis. 2015; 36(2):91-101. [DOI:10.1027/0227-5910/a000305] [PMID]
[6] Davidson JE, Stuck AR, Zisook S, Proudfoot J. Testing a strategy to identify incidence of nurse suicide in the United States. Journal of Nursing Administration. 2018; 48(5):259-65. [DOI:10.1097/NNA.0000000000000610] [PMID]

[7] Hawton K, Vislisel L. Suicide in nurses. Suicide and Life Threatening Behavior. 1999; 29(1):86-95. [PMID]

[8] Kõlves K, De Leo D. Suicide in medical doctors and nurses: An analysis of the queens land suicide register. The Journal of Nervous and Mental Disease. 2013; 201(11):987-90. [DOI:10.1097/ NMD.0000000000000047] [PMID]

[9] Osafo J, Akotia CS, Boakye KE, Dickon E. Between moral infraction and existential crisis: Exploring physicians and nurses' attitudes to suicide and the suicidal patient in Ghana. International Journal of Nursing Studies. 2018; 36(2):91-101. [DOI:10.1016/j. ijnurstu.2018.05.017] [PMID]

[10] Hawton K, Agerbo E, Simkin S, Platt B, Mellanby RJ. Risk of suicide in medical and related occupational groups: A national study based on Danish case population-based registers. Journal of Affective Disorders. 2011; 134(1-3):320-6. [DOI:10.1016/j. jad.2011.05.044] [PMID]

[11] Van Orden KA, Witte TK, Cukrowicz KC, Braithwaite SR, Selby EA, Joiner Jr TE. The interpersonal theory of suicide. Psychological Review. 2010;117(2):575-600. [DOI:10.1037/a0018697] [PMID] [PMCID]

[12] Hoshmandja, M., Javanmard, A., Marashi, S. [The relationship between Self-Efficacy, cognitive meta cognitive strategies with academic achievement among students high school boys nomads of Fars Province (Persian)]. Technology of Education. 2014; 8(3):181-71

[13] Boroumand, A. [Chronic pain, pain Self-Efficacy and Suicidal Ideation: The moderating role of pain Self-Efficacy on relation between depression and Suicidal Ideation in chronic pain patients (Persian)]. Journal of Fundamentals of Mental Health. 2012; 14(54):63-152

[14] Kobayashi Y, Fujita K, Kaneko Y, Motohashi Y. Self-Efficacy as a Suicidal Ideation predictor: A population cohort study in rural Japan. Open Journal of Preventive Medicine. 2015; 5(2):6171. [DOI:10.4236/ojpm.2015.52007]

[15] Ammentorp J, Sabroe S, Kofoed PE, Mainz J. The effect of training in communication skills on medical doctors' and nurses' Self-Efficacy: A randomized controlled trial. Patient Education and Counseling. 2007; 66(3):270-7. [DOI:10.1016/j. pec.2006.12.012] [PMID]

[16] Yang YK. A study on burnout, emotional labor, and Self-Efficacy in nurses. Journal of Korean Academy of Nursing Administration. 2011; 17(4):423-31. [DOI:10.11111/jkana.2011.17.4.423]

[17] Ravanipour M, Ahmadian A, Yazdanpanah A, Soltanian A R. [Assessing the relationship between Self-Efficacy and clinical decision-making in hospital nurse (Persian)]. Scientific Journal of Hamadan Nursing \& Midwifery Faculty. 2015; 23(4):77-86.

[18] Mohammadi A, Kheftan P, Amirpour B, Sepidehdam MA, Gholami Jam F. [Self-Efficacy relationship with social health among nurses in Taleghani Hospital in Kermanshah (Persian)]. Iranian Journal of Health Education and Health Promotion 2018; 6(1):72-9. [DOI:10.30699/acadpub.ijhehp.6.1.72]

[19] Hosseini MA, Azimzadeh E. [Correlation between Self-Efficacy and nurses' conflict management strategies (Persian)]. Journal of Health Promotion Management. 2013; 2(4):16-23. 
[20] Brody S, Carson CM. Brief report: Self-harm is associated with immature defence mechanisms but not substance use in a nonclinical Scottish adolescent sample. Journal of Adolescence. 2012; 35(3):765-7. [DOI:10.1016/j.adolescence.2011.09.001] [PMID]

[21] Plutchik R, Botsis AJ, Van Praag HM. Psychopathology, selfesteem, sexual and ego functions as correlates of suicide and violence risk. Archives of Suicide Research. 1995; 1(1):27-38. [DOI:10.1080/13811119508258972]

[22] Apter A, Gothelf D, Offer R, Ratzoni G, Orbach I, Tyano S, et al. Suicidal adolescents and ego defense mechanisms. Journal of the American Academy of Child \& Adolescent Psychiatry. 1997; 36(11):1520-7. [DOI:10.1016/S0890-8567(09)66560-6] [PMID]

[23] Yang YK. Effects of ego-resilience and work environment on job satisfaction in psychiatric nurses. Journal of Korean Academy of Psychiatric and Mental Health Nursing. 2015; 24(4):226-35. [DOI:10.12934/jkpmhn.2015.24.4.226]

[24] Lee OS, Gu MO, Kim MJ. Influence of emotional intelligence and ego resilience on interpersonal relationship of nurses. Journal of the Korea Academia-Industrial Cooperation Society. 2015; 16(6):3902-10. [DOI:10.5762/KAIS.2015.16.6.3902]

[25] Markstrom CA, Sabino VM, Turner BJ, Berman RC. The psychosocial inventory of Ego Strengths: Development and validation of a new Eriksonian measure. Journal of Youth and Adolescence. 1997; 26(6):705-32. [DOI:10.1023/ A:1022348709532]

[26] Gold SN. Relations between level of ego development and adjustment pattern in adolescents. Journal of Personality Assessment. 1980; 44(6):630-8. [DOI:10.1207/s15327752jpa4406_11] [PMID]

[27] Van Orden KA. Construct validity of the interpersonal needs questionnaire. Tallahassee, Florida: The Florida State University; 2009.

[28] Hill RM, Rey Y, Marin CE, Sharp C, Green KL, Pettit JW. Evaluating the interpersonal needs questionnaire: Comparison of the reliability, factor structure, and predictive validity across five versions. Suicide and Life-Threatening Behavior. 2015; 45(3):30214. [DOI:10.1111/sltb.12129] [PMID]

[29] Rashid S, Kiani Chalmari A, Yeganeh N, Zeinaddini S. [The relationship between death anxiety, mattering, Perceived Burdensomeness and Thwarted Belongingness with suicidal behavior in college students (The interpersonal-psychological theory for suicide) (Persian)]. Iranian Journal of Emergency Care. 2017; 1(1):32-42.

[30] Sherer M, Maddux JE, Mercandante B, Prentice-Dunn S, Jacobs B, Rogers RW. The Self-Efficacy Scale: Construction and validation. Psychological Reports. 1982; 51(2):663-71. [DOI:10.2466/ pr0.1982.51.2.663]

[31] Anisi J, Fathiashteiani A, Salimi H, Ahmadinoodeh K. [Assessment of Beck scale of suicide thoughts in soldiers (Persian)]. Journal of Military Medicine. 2005; 23(7):33-7.

[32] Bryan CJ. The clinical utility of a brief measure of Perceived Burdensomeness and Thwarted Belongingness for the detection of suicidal military personnel. Journal of Clinical Psychology. 2011; 67(10):981-92. [DOI:10.1002/jclp.20726] [PMID]

[33] O'Keefe VM, Wingate LR, Tucker RP, Rhoades-Kerswill S, Slish ML, Davidson CL. Interpersonal suicide risk for American Indians: Investigating Thwarted Belongingness and Perceived Burdensomeness. Cultural Diversity and Ethnic Minority Psychology. 2014; 20(1):61-7. [DOI:10.1037/a0033540] [PMID]
[34] Hill RM, Pettit JW. Suicidal Ideation and sexual orientation in college students: The roles of Perceived Burdensomeness, Thwarted Belongingness, and perceived rejection due to sexual orientation. Suicide and Life-Threatening Behavior. 2012 42(5):567-79. [DOI:10.1111/j.1943-278X.2012.00113.x] [PMID]

[35] Nabavi SS, Sohrabi F, Afrooz G, Delavar A, Hosseinian S. [Relationship between Self-Efficacy and mental health among teachers: The role of perceived social support (Persian)]. Journal of Research in Psychological Health. 2017; 11(2):50-68. [DOI:10.29252/ rph.11.2.50]

[36] Valois RF, Zullig KJ, Hunter AA. Association between adolescent suicide ideation, suicide attempts and emotional SelfEfficacy. Journal of Child and Family Studies. 2015; 24(2):237-48. [DOI:10.1007/s10826-013-9829-8] 